\title{
PROPAGATION PHENOMENA FOR MONO-WEAKLY HYPONORMAL OPERATOR PAIRS
}

\author{
Yonguiang Duan, Shihao Pang And SiYu Wang
}

Abstract. In this note, we strengthen some of flatness results for mono-polynomially hyponormal and mono-weakly 2 -hyponormal 2 -variable weighted shifts in $[15,16,17]$.

Mathematics subject classification (2010): 47B20, 47B37, 47A13.

Keywords and phrases: 2-variable weighted shifts, flatness, $k$-hyponormal, subnormal, mono-weakly $k$-hyponormal, mono-polynomially hyponormal.

\section{REFERENCES}

[1] J. AGLER, The Arveson extension theorem and coanalytic models, Integral Equations Operator Theory 5 (1982), no. 5, 608-631.

[2] A. Athavale, On joint hyponormality of operators, Proc. Amer. Math. Soc. 103 (1988), no. 2, 417 423.

[3] J. Conway, The Theory of Subnormal Operators, Mathematical Surveys and Monographs, vol. 36, American Mathematical Society, Providence, RI, 1991.

[4] Y. CHOI, A propagation of the quadratically hyponormal weighted shifts, Bull. korean math. soc. 37 (2000) 347-352.

[5] R. CURTO, Quadratically hyponormal weighted shifts, Integral Equations Operator Theory 13 (1990) 49-66.

[6] R. CuRTO AND L. Fialkow, Recursively generated weighted shifts and the subnormal completion problem, II, Integral Equations Operator Theory 18 (1994) 369-426.

[7] R. Curto, S. LeE AND J. Yoon, $k$-Hyponormality of multivariable weighted shifts, J. Funct. Anal. 229 (2005) 462-480.

[8] R. CuRTO, S. LeE And J. Yoon, Which 2-hyponormal 2-variable weighted shifts are subnormal?, Linear Algebra Appl. 429 (2008), 2227-2238.

[9] R. CuRto, P. Muhly And J. XiA, Hyponormal pairs of commuting operators, Oper. Theory: Adv. Appl. 35 (1998) 1-22.

[10] R. Curto AND J. Yoon, Propagation phenomena for hyponormal 2-variable weighted shifts, J. Operator Theory 58 (2007) 175-203.

[11] R. CURTO And M. PUtinar, Nearly subnormal operators and moment problems, J. Funct. Anal. 115 (1993), no. 2, 480-497.

[12] Y. DUAN, T. QI, Weakly k-hyponormal and polynomially hyponormal commuting operator pairs, Sci. China Math. 58 (2015) 405-422.

[13] P. FAN, A note on hyponormal weighted shifts, Proc. Amer. Math. Soc. 92 (1984), 271-272.

[14] A. Jos HI, Hyponormal polynomials of monotone shifts, Ph. D. dissertation, Purdue University, 1971.

[15] J. Kim AND J. Yoon, Flat phenomena of 2-variable weighted shifts, Linear Algebra Appl. 486 (2015) 234-254.

[16] J. Kim And J. Yoon, Hyponormality for commuting pairs of operators, J. Math. Anal. Appl. 434 (2016) 1077-1090.

[17] J. Kim And J. Yoon, Properties of mono-weakly hyponormal 2-variable weighted shifts, Linear Multilinear Algebra 65 (2017) 1260-1275.

[18] P. Halmos, Normal dilations and extensions of operators, Summa Brasil. Math. 2 (1950), 125-134. 
[19] C. Li AND J. AHn, On the flatness of semi-cubically hyponormal weighted shifts, Kyungpook Math. J. 48 (2008), 721-727.

[20] S. McCullough And V. Paulsen, A note on joint hyponormality, Proc. Amer. Math. Soc. 107 (1989), 187-195.

[21] J. STAMPFLI, Which weighted shifts are subnormal, Pacific J. Math. 17 (1966), 367-379.

[22] A. ShIELDS, Weighted Shift Operator and Analytic Function Theory, Math Surveys, vol. 13. Amer. Math. Soc., Providence, 1974. 